PROCEEDINGS OF THE

AMERICAN MATHEMATICAL SOCIETY

Volume 127, Number 6, Pages 1761-1770

S 0002-9939(99)04771-1

Article electronically published on February 11, 1999

\title{
SUPPORT FUNCTIONALS AND SMOOTH POINTS IN ABSTRACT $M$ SPACES
}

\author{
WANG BAOXIANG AND WANG TINGFU
}

(Communicated by Palle E. T. Jorgensen)

\begin{abstract}
By presenting some properties of support functionals in abstract $M$ spaces, we get some sufficient and necessary conditions for smooth points in abstract $M$ (function) spaces. Moreover, the notion of the smallest support semi-norm is introduced and an explicit form for this functional in abstract $M$ function spaces is also given.
\end{abstract}

\section{INTRODUCTION}

There are some recent papers devoted to the study of support functionals and their applications in a class of Banach function spaces. Hudzik and Ye in [3] gave a characteristic of support functionals in Musielak-Orlicz sequence spaces equipped with the Luxemburg norm, where they introduced a generalized Banach limit to describe singular support functionals. Some characteristics of support functionals in Orlicz spaces endowed with the Luxemburg norm and with the Orlicz norm have been obtained by Wang in [9] and Chen, Hudzik and Kaminska in [1], respectively. Moreover, some criteria of smooth points and exposed points in these spaces were also considered in [1], [3] and [9].

The aim of the present paper is to study the support functionals and their applications in abstract $M$ spaces and in abstract $M$ function spaces. This paper is organized as follows. The first part is an introduction. The second part consists of some results concerning the characteristic of support functionals in abstract $M$ spaces. Section 3 is devoted to some applications of support functionals in these spaces. Some criteria of smooth points are obtained. In section 4 we further discuss the support functionals at a point in abstract $M$ function spaces. The notion of the smallest support semi-norm at a point is introduced and an explicit form for the smallest support semi-norm at any point in such spaces is given.

Let $X$ be a normed linear space and $X^{*}$ be its dual space. $f \in X^{*}$ is said to be a support functional at $x \in X \backslash\{0\}$ if $\langle f, x\rangle=\|x\|$ and $\|f\|=1$. For convenience, we denote by $S_{x}^{*}$ the set of all support functionals at $x$. We say that $x \in X \backslash\{0\}$ is a smooth point if the support functional at $x$ is unique.

Let $X$ be a normed Riesz space. The norm on $X$ is said to be $\infty$-additive if $x, y \in X$ with $x \wedge y=0$ implies that $\|x+y\|=\max (\|x\|,\|y\|)$. A normed

Received by the editors September 23, 1996 and, in revised form, September 16, 1997.

1991 Mathematics Subject Classification. Primary 46B20; Secondary 46E30.

Key words and phrases. Abstract $M$ (function) space, support functional, the smallest support semi-norm, smooth point. 
Riesz space $X$ equipped with the $\infty$-additive norm is called an abstract $M$ space $(X \in A M)^{1}$ (cf. [7] and [13]).

Let $(\Omega, \Sigma, \mu)$ be a complete $\sigma$-finite measure space. A normed space $X$ consisting of equivalence classes, modulo equality almost everywhere, of real valued measurable functions on $\Sigma$ is called a normed Köthe function space, if $|x(t)| \leq|y(t)|$ a.e. on $\Omega$, with $x$ measurable and $y \in X$ implies that $x \in X$ and $\|x\| \leq\|y\|$ (cf. [6]). A normed Köthe function space equipped with the $\infty$-additive norm is called an abstract $M$ function space $(X \in A M F)$.

\section{Some properties of Support functionals in $A M$ AND $A M F$ SPACES}

Proposition 2.1. Let $X \in A M$ and $x \in X \backslash\{0\}$. Let $f \in S_{x}^{*}$. Then we have

(i) $\langle f, y\rangle \geq 0$ if $x^{-} \wedge y=0$ or $x^{+} \wedge(-y)=0$;

(ii) $\langle f, y\rangle \leq 0$ if $x^{-} \wedge(-y)=0$ or $x^{+} \wedge y=0$;

(iii) $\langle f, y\rangle=0$ if $|x| \wedge|y|=0$.

Proof. First, we consider case (ii). If $x^{-} \wedge(-y)=0$, then $y \leq 0$. Suppose that $Q \leq-1$ satisfying $\|y / Q\| \leq\|x\|$. Noticing that $y / Q \geq 0$ and

$$
\begin{aligned}
|x-y / Q| & =\left|x^{+}-x^{-}-y / Q\right| \\
& =x^{+} \vee\left(x^{-}+y / Q\right)-x^{+} \wedge\left(x^{-}+y / Q\right) \leq x^{+} \vee\left(x^{-}+y / Q\right),
\end{aligned}
$$

we have

$$
\|x-y / Q\| \leq \max \left(\left\|x^{+}\right\|,\left\|x^{-}\right\|,\|y / Q\|\right)=\|x\| .
$$

Thus

$$
\langle f, x-y / Q\rangle \leq\|f\|\|x\|=\langle f, x\rangle .
$$

It follows that $\langle f, y / Q\rangle \geq 0$. So $\langle f, y\rangle \leq 0$.

Similarly, if $x^{+} \wedge y=0$, we can choose $R \geq 1$ such that $\|y / R\| \leq\|x\|$. We have $|x+y / R| \leq x^{-} \vee\left(x^{+}+y / R\right)$. It follows that $\|x+y / R\| \leq\|x\|$. So we have $\langle f, x+y / R\rangle \leq\langle f, x\rangle$, which implies that $\langle f, y\rangle \leq 0$.

Next, if we replace $y$ with $-y$ in the above procedure, one can easily verify that (i) holds.

Finally, (iii) is a direct consequence of (i) and (ii).

Proposition 2.2. Let $X \in A M$ and $x \in X \backslash\{0\}$. Let $f \in S_{x}^{*}$. Suppose that $|x|=x_{1} \vee x_{2}$ and $x_{1} \wedge x_{2}=0$. If $\left\|x_{1}\right\|<\|x\|$, then $\langle f, y\rangle=0$ for all $y \in X$ satisfying $|y| \wedge x_{2}=0$.

Proof. First, we prove that $\left\langle f, x_{1} \wedge x^{+}\right\rangle=\left\langle f, x_{1} \wedge x^{-}\right\rangle=0$. It follows from Proposition 2.1 that $\left\langle f, x_{1} \wedge x^{+}\right\rangle \geq 0$ and $\left\langle f, x_{1} \wedge x^{-}\right\rangle \leq 0$. We now show that $\left\langle f, x_{1} \wedge x^{+}\right\rangle=0$. Assume for the contrary that $\left\langle f, x_{1} \wedge x^{+}\right\rangle>0$. Let $a>1$ satisfy $\left\|a x_{1}\right\|=\|x\|$. Put

$$
z=x+(a-1)\left(x^{+} \wedge x_{1}\right) .
$$

Noticing that $x^{+}=|x| \wedge x^{+}=x^{+} \wedge x_{1}+x^{+} \wedge x_{2}$, we have

$$
z=x^{+}-x^{-}+(a-1)\left(x^{+} \wedge x_{1}\right)=a\left(x^{+} \wedge x_{1}\right)+x^{+} \wedge x_{2}-x^{-} .
$$

\footnotetext{
${ }^{1}$ We do not need that $X$ is a Banach lattice.
} 
It follows that $\|z\| \leq \max \left(\left\|a\left(x^{+} \wedge x_{1}\right)\right\|,\left\|x^{+} \wedge x_{2}\right\|,\left\|x^{-}\right\|\right) \leq\|x\|$. On the other hand,

$$
\langle f, z\rangle=\langle f, x\rangle+(a-1)\left\langle f, x^{+} \wedge x_{1}\right\rangle>\langle f, x\rangle .
$$

This contradicts the inequality $\langle f, z\rangle \leq\|f\|\|z\| \leq\|x\|=\langle f, x\rangle$. Similarly, we can prove that $\left\langle f, x_{1} \wedge x^{-}\right\rangle=0$. Hence we have

$$
\langle f, x\rangle=\left\langle f, x^{+} \wedge x_{2}-x^{-} \wedge x_{2}\right\rangle=\|x\| .
$$

Next, assume that $|y| \wedge x_{2}=0$. We can choose $Q \geq 1$ such that $\|y / Q\| \leq\|x\|$. Let

$$
z=x_{2} \wedge x^{+}-x_{2} \wedge x^{-}+y / Q
$$

It is easy to see that $\|z\| \leq\|x\|$. We have

$$
\begin{aligned}
\langle f, z\rangle & =\left\langle f, x_{2} \wedge x^{+}-x_{2} \wedge x^{-}\right\rangle+\langle f, y / Q\rangle \\
& =\langle f, x\rangle+\langle f, y / Q\rangle=\|x\|+\langle f, y / Q\rangle .
\end{aligned}
$$

This implies that $\langle f, y\rangle \leq 0$. Replacing $y$ with $-y$ in the above procedure, we see that $\langle f,-y\rangle \leq 0$. So we have $\langle f, y\rangle=0$.

In view of Propositions 2.1 and 2.2, we have

Corollary 2.3. Let $X \in A M F$, and let $x \in X \backslash\{0\}$ and $f \in S_{x}^{*}$. Then for any $y \in X$, we have

(i) $\langle f, y\rangle \geq 0$ if $x(t) y(t) \geq 0$ a.e. on $\Omega$;

(ii) $\langle f, y\rangle \leq 0$ if $x(t) y(t) \leq 0$ a.e. on $\Omega$;

(iii) $\langle f, y\rangle=0$ if $x(t) y(t)=0$ a.e. on $\Omega$.

Corollary 2.4. Let $X \in A M F$, and let $x \in X \backslash\{0\}$ and $f \in S_{x}^{*}$. Suppose that $e \in \Sigma$ and $\left\|x \chi_{e}\right\|<\|x\|$. Then we have $\left\langle f, y \chi_{e}\right\rangle=0$ for any $y \in X$.

\section{Smooth points in $A M$ and $A M F$ spaces}

Let $X$ be a normed Riesz space. We say that the element $x \neq 0$ in $X$ is an atom if it follows from $x=y+z$ and $|y| \wedge|z|=0$ that $y=0$ or $z=0$.

Lemma 3.1. Let $x \in A M$ and let $x \in X \backslash\{0\}$. If there exist $x_{1}, x_{2} \in X$ such that $\left|x_{1}\right| \wedge\left|x_{2}\right|=0, x_{1}+x_{2}=x$ and $\left\|x_{1}\right\|=\left\|x_{2}\right\|=\|x\|$, then $S_{x}^{*}$ contains at least two elements.

Proof. Let $f_{i}$ be a support functional at $x_{i}$ for $i=1,2$. In view of Propositions 2.1 and 2.2 , we have $\left\langle f_{i}, x_{j}\right\rangle=0$ for $i, j=1,2, i \neq j$. It follows that

$$
\left\langle f_{i}, x\right\rangle=\left\langle f_{i}, x_{i}\right\rangle=\left\|x_{i}\right\|=\|x\| .
$$

for $i=1,2$. Thus $f_{1}$ and $f_{2}$ are both the support functionals at $x$. Clearly, $f_{1} \neq f_{2}$. The proof is complete.

Theorem 3.2. Let $X \in A M$ have an order complete and order semi-continuous norm. Let $x \in X \backslash\{0\}$. Then $x$ is a smooth point if and only if there exists an atom $y \in X$ such that $x=y+z,|y| \wedge|z|=0,\|y\|=\|x\|$ and $\|z\|<\|x\|$. 
Proof. Necessity. Let $x \in X$ be a smooth point. It follows that $\min \left(\left\|x^{+}\right\|,\left\|x^{-}\right\|\right)<$ $\|x\|$. Indeed, if $\left\|x^{+}\right\|=\left\|x^{-}\right\|=\|x\|$, in view of Lemma 3.1 there exist at least two support functionals at $x$. This contradicts that $x$ is smooth. $X \in A M$ implies that $\max \left(\left\|x^{+}\right\|,\left\|x^{-}\right\|\right)=\|x\|$. We may assume without loss of generality that $\left\|x^{+}\right\|=\|x\|$. Put

$$
A=\left\{w_{\alpha}: 0 \leq w_{\alpha} \leq x^{+},\left\|w_{\alpha}\right\|<\|x\| \text { and }\left(x^{+}-w_{\alpha}\right) \wedge w_{\alpha}=0\right\} .
$$

It is easy to see that $w_{\alpha_{i}} \in A(i=1,2)$ implies $w_{\alpha_{1}} \vee w_{\alpha_{2}} \in A$. It follows that $A$ is an upwards directed set. $X$ is order complete, so we have $w_{\alpha} \uparrow \bigvee_{\alpha} w_{\alpha}$. For convenience, we denote $w=\bigvee_{\alpha} w_{\alpha}$. Since $X$ has the order semi-continuous norm, we have $\|w\|=\sup _{\alpha}\left\|w_{\alpha}\right\|$. Therefore, there exists a sequence $\left\{w_{\alpha_{n}}\right\} \subset A$ such that $\left\|w_{\alpha_{n}}\right\| \geq\|w\|-1 / n$. We may assume without loss of generality that $w_{\alpha_{n}} \uparrow$. Indeed, we can select $\bigvee_{i<n} w_{\alpha_{i}}$ instead of $w_{\alpha_{n}}$. It follows that $\left\|w_{\alpha_{n}}\right\| \uparrow\|w\|$. This implies that $\|w\|<\left\|x^{+}\right\|$. In fact, if $\|w\|=\left\|x^{+}\right\|=\|x\|$, then we have $\left\|w_{\alpha_{n}}\right\| \uparrow\left\|x^{+}\right\|$. Put $v_{0}=w_{\alpha_{1}}$ and $v_{n}=w_{\alpha_{n+1}}-w_{\alpha_{n}}(n \geq 1)$. We can easily see that $w_{\alpha_{n}}=\bigvee_{0 \leq i \leq n} v_{i}$ and $v_{i} \wedge v_{j}=0(i \neq j)$. It follows from $X \in A M$ that there exists a subsequence of $\left\{v_{n}\right\}$, still denoted by $\left\{v_{n}\right\}$ such that $\left\|v_{n}\right\| \uparrow\left\|x^{+}\right\|$. Let $w_{1}=\bigvee_{k \geq 1} v_{2 k+1}$ and $w_{2}=\bigvee_{k>1} v_{2 k}$. We have $\left\|w_{1}\right\|=\left\|w_{2}\right\|=\left\|x^{+}\right\|$and $w_{1} \wedge w_{2}=0$. In virtue of Lemma 3.1 we can see that there exist at least two support functionals at $x$. This contradicts that $x$ is smooth.

Let $y=x^{+}-w$. It follows from $w_{\alpha} \wedge\left(x-w_{\alpha}\right)=0$ that $w \wedge\left(x^{+}-w\right)=0$ (cf. [8], §15). $X \in A M$ and $\|w\|<\left\|x^{+}\right\|$imply that $\|y\|=\left\|x^{+}\right\|=\|x\|$. Now we show that $y$ is an atom. If not, then $y=y_{1}+y_{2}, y_{1} \wedge y_{2}=0$ and $y_{1}, y_{2}>0$. By Lemma 3.1 we have $\min \left(\left\|y_{1}\right\|,\left\|y_{2}\right\|\right)<\left\|x^{+}\right\|$. Assume that $\left\|y_{1}\right\|<\left\|x^{+}\right\|$. One can easily see that $w \vee y_{1} \in A$. This contradicts the fact $w=\bigvee_{\alpha} w_{\alpha}$. Let $z=w-x^{-}$. It follows that $x=y+z,|y| \wedge|z|=0$ and $\|z\|<\|x\|$.

Sufficiency. Let $x=y+z, y$ be an atom satisfying $\|y\|=\|x\|,\|z\|<\|x\|$ and $|y| \wedge|z|=0$. Let $f$ be a support functional at $x$. It follows from Proposition 2.2 that $\langle f, z\rangle=0$. So $f$ is also a support functional at $y$. Clearly, $y$ has a unique support functional. Indeed, we first have $y<0$ or $y>0$ (cf. [8], §26). Assume without loss of generality that $y>0$. For any $x \in X^{+}$, let

$$
B=\{u: 0 \leq u \leq x, u \wedge(x-u)=0, \text { and } u \wedge y=0\} .
$$

Similarly to the above, we see that $B$ is an upwards directed set and so, $u_{0} \stackrel{\text { def }}{=}$ $\sup _{u \in B} u$ exists. If $u_{0} \neq x$, we claim that $x-u_{0}$ is also an atom. If not, then there exist $u_{1}$ and $u_{2}$ such that $x-u_{0}=u_{1} \vee u_{2}$ and $u_{1} \wedge u_{2}>0$. Since $y$ is an atom, we have $u_{1} \wedge y=0$ or $u_{2} \wedge y=0$. We may assume that $u_{1} \wedge y=0$. One can easily verify that $u_{0} \vee u_{1} \in B$. A contradiction. It follows from Theorem 26.4 in [8] that if $u_{0} \neq x$, then either $\left(x-u_{0}\right) \wedge y=0$ or $\left(x-u_{0}\right)=a y$ for some $a \in(0,1]$. Thus, for any $f_{i} \in S_{y}^{*}(i=1,2)$, from the equality

$$
\left\langle f_{i}, x\right\rangle=\left\langle f_{i}, u_{0}\right\rangle+\left\langle f_{i}, x-u_{0}\right\rangle=a, \quad i=1,2,
$$

we see that $\left\langle f_{i}, x\right\rangle=0$ if $u_{0}=x$ or $\left(x-u_{0}\right) \wedge y=0$, and $\left\langle f_{i}, x\right\rangle=a$ if $\left(x-u_{0}\right)=a y$. This leads to $f_{1} \equiv f_{2}$. Thus, the support functional at $y$ is unique. The same result also holds true for $x \in X^{-}$and so, for $x \in X$. The proof is complete.

Theorem 3.3. Let $X \in A M F$ and let $x \in X \backslash\{0\}$. Then $x$ is a smooth point if and only if for any disjoint $e_{1}, e_{2} \in \Sigma, \min \left(\left\|x \chi_{e_{1}}\right\|,\left\|x \chi_{e_{2}}\right\|\right)<\|x\|$. 
Proof. Sufficiency. We assume without loss of generality that $\|x\|=1$. If $x$ is not a smooth point, then there exist two elements $f_{1}$ and $f_{2}$ in $S_{x}^{*}$ and $y \in X,\|y\| \leq 1$ such that

$$
\left\langle f_{1}, y\right\rangle \neq\left\langle f_{2}, y\right\rangle
$$

Let

$$
\begin{aligned}
& \Omega_{0}=\{t \in \Omega: x(t) y(t)=0\}, \\
& \Omega_{1}=\{t \in \Omega: x(t) y(t)>0\}, \\
& \Omega_{2}=\{t \in \Omega: x(t) y(t)<0\} .
\end{aligned}
$$

It follows from the hypothesis that there exists only one $\Omega_{j}$ such that $\left\|x \chi_{\Omega_{j}}\right\|=1$ for some $j \in\{0,1,2\}$. We shall show that this yields a contradiction. Let us consider for this purpose three separate cases.

Case I. $\left\|x \chi_{\Omega_{0}}\right\|=1$. We have $\left\|x \chi_{\Omega_{i}}\right\|<1$ for $i=1,2$. In virtue of Corollaries 2.3 and 2.4, we have $\langle f, y\rangle=0$ for any $f \in S_{x}^{*}$, which implies that $\left\langle f_{1}, y\right\rangle=\left\langle f_{2}, y\right\rangle=0$. A contradiction.

Case II. $\left\|x \chi_{\Omega_{1}}\right\|=1$. We have $\left\|x \chi_{\Omega_{i}}\right\|<1$ for $i=0,2$. First, we assume that $x(t), y(t)>0$ for all $t \in \Omega_{1}$. Take an arbitrary $\varepsilon>0$ and let

$$
\Omega_{1 \varepsilon}=\left\{t \in \Omega_{1}: y(t)<(1+\varepsilon) x(t)\right\} .
$$

We have $\left\|x \chi_{\Omega \backslash \Omega_{1 \varepsilon}}\right\|<1$. Indeed, if this inequality does not hold, then $\left\|y \chi_{\Omega \backslash \Omega_{1 \varepsilon}}\right\| \geq$ $1+\varepsilon$. A contradiction. So we have $\left\|x \chi_{\Omega_{1 \varepsilon}}\right\|=1$. Let

$$
\begin{aligned}
& \Omega_{>}=\left\{t \in \Omega_{1 \varepsilon}: 1 \leq \frac{y(t)}{x(t)}<1+\varepsilon\right\}, \\
& \Omega_{<}=\left\{t \in \Omega_{1 \varepsilon}: 0 \leq \frac{y(t)}{x(t)}<1\right\} .
\end{aligned}
$$

It follows that $\max \left(\left\|x \chi_{\Omega_{>}}\right\|,\left\|x \chi_{\Omega_{<}}\right\|\right)=1$. We will divide Case II into the following two cases.

Case IIa. $\left\|x \chi_{\Omega_{>}}\right\|=1$. We have $\left\|x \chi_{\Omega_{<}}\right\|<1$. Noticing that

$$
\Omega=\left(\Omega_{1} \backslash \Omega_{1 \varepsilon}\right) \cup \Omega_{<} \cup\left(\Omega \backslash \Omega_{1}\right) \cup \Omega_{>} \quad \text { and } \quad\left\|x \chi_{\left(\Omega \backslash \Omega_{1}\right) \cup\left(\Omega_{1} \backslash \Omega_{1 \varepsilon}\right) \cup \Omega_{<}}\right\|<1 \text {, }
$$

we obtain that for any $f \in S_{x}^{*}$,

$$
\langle f, y\rangle=\left\langle f, y \chi_{\Omega_{>}}\right\rangle+\left\langle f, y \chi_{\left(\Omega \backslash \Omega_{1}\right) \cup\left(\Omega_{1} \backslash \Omega_{1 \varepsilon}\right) \cup \Omega_{<}}\right\rangle=\left\langle f, y \chi_{\Omega_{>}}\right\rangle
$$

and

$$
\langle f, x\rangle=\left\langle f, x \chi_{\Omega_{>}}\right\rangle .
$$

Since $t \in \Omega_{>}$implies that $x(t) \leq y(t)<(1+\varepsilon) x(t)$, we have

$$
\begin{aligned}
1 & =\left\langle f, x \chi_{\Omega_{>}}\right\rangle \leq\left\langle f, y \chi_{\Omega_{>}}\right\rangle \\
& =\langle f, y\rangle \leq(1+\varepsilon)\left\langle f, x \chi_{\Omega_{>}}\right\rangle=1+\varepsilon .
\end{aligned}
$$

Since $\varepsilon>0$ is arbitrary, this means that $\langle f, y\rangle=1$. Thus $\left\langle f_{1}, y\right\rangle=\left\langle f_{2}, y\right\rangle=1$. This is a contradiction.

Case IIb. $\left\|x \chi_{\Omega_{<}}\right\|=1$. We have $\left\|x \chi_{\Omega_{>}}\right\|<1$. Put

$$
e_{i}=\left\{t \in \Omega_{<}: \frac{i-1}{2^{n}} \leq \frac{y(t)}{x(t)}<\frac{i}{2^{n}}\right\}, \quad i=1, \ldots, 2^{n} .
$$

It is easy to see that there exists only one $e_{i_{n}} \subset \Omega_{<}, i \in\left\{1,2, \ldots, 2^{n}\right\}$, such that $\left\|x \chi_{e_{i_{n}}}\right\|=1$, i.e. $\left\|x \chi_{e_{i}}\right\|<1$ for any $i \neq i_{n}, 1 \leq i \leq 2^{n}$. Moreover, we have 
$e_{i_{n+1}} \subset e_{i_{n}}$. In fact, if $e_{i_{n+1}} \subset e_{i_{n}^{\prime}}$ for some $i_{n}^{\prime} \in\left\{1, \ldots, 2^{n}\right\}$ and $i_{n}^{\prime} \neq i_{n}$, then $\left\|x \chi_{e_{i_{n}^{\prime}}}\right\|=1$. This contradicts the equality $\left\|x \chi_{e_{i_{n}}}\right\|=1$. So we have obtained that

$$
\langle f, z\rangle=\left\langle f, z \chi_{e_{i_{n}}}\right\rangle
$$

for all $f \in S_{x}, z \in X$ and $n \in \mathbb{N}$. Therefore

$$
\begin{aligned}
\frac{i_{n}-1}{2^{n}} & =\frac{i_{n}-1}{2^{n}}\left\langle f, x \chi_{e_{i_{n}}}\right\rangle \leq\left\langle f, y \chi_{e_{i_{n}}}\right\rangle \\
& =\langle f, y\rangle \leq \frac{i_{n}}{2^{n}}\left\langle f, x \chi_{e_{i_{n}}}\right\rangle=\frac{i_{n}}{2^{n}} .
\end{aligned}
$$

Clearly, $\left\{i_{n} / 2^{n}\right\}$ is a Cauchy sequence. Suppose that $\lim _{n \rightarrow \infty} i_{n} / 2^{n}=a$. Then we have $\langle f, y\rangle=a$ for any $f \in S_{x}^{*}$. This implies that $\left\langle f_{1}, y\right\rangle=\left\langle f_{2}, y\right\rangle$. A contradiction.

At the end of Case II, we consider the general case $x(t) y(t)>0$ for all $t \in \Omega_{1}$. Let $f \in S_{x}^{*}$ and let $f^{\prime}$ satisfy

$$
\left\langle f^{\prime}, z \operatorname{sign}(x)\right\rangle=\langle f, z\rangle
$$

for any $z \in X$. One can easily verify that $f \in S_{x}^{*}$ if and only if $f^{\prime} \in S_{|x|}^{*}$. Similarly to the above discussions, we can prove that $\left\langle f_{1}^{\prime}, y \operatorname{sign}(x)\right\rangle=\left\langle f_{2}^{\prime}, y \operatorname{sign}(x)\right\rangle$, which implies that $\left\langle f_{1}, y\right\rangle=\left\langle f_{2}, y\right\rangle$. A contradiction.

Case III. $\left\langle x \chi_{\Omega_{2}}\right\rangle=1$. In exactly the same way as in Case II, we can prove the result.

Necessity. It is a direct consequence of Lemma 3.1. The proof is finished.

Remark 3.4. By Theorems 3.2 and 3.3 we have seen that $L^{\infty}(\Omega, \Sigma, \mu)$ has no smooth point if $(\Omega, \Sigma, \mu)$ is an atomless measure space and $x \in l^{\infty} \backslash\{0\}$ is a smooth point if and only if $\sup _{i \in N}|x(i)|$ is attainable at only one coordinate and lim $\sup _{i \rightarrow \infty}|x(i)|<$ $\|x\|_{l^{\infty}}$. So we have revised the criteria of smooth points in $l^{\infty}$ given by [12].

At the end of this section, we give some counterexamples to show that the order complete and order semi-continuous norm in Theorem 3.2 are necessary for criterion of a smooth point.

Example 3.5. Let $\phi \in l^{\infty *}$ be a positive singular functional and an extreme point of the unit ball of $l^{\infty *}$. In view of Theorem 9 in [2] we have $\langle\phi, x\rangle\langle\phi, y\rangle=0$ for all $x, y \in l^{\infty}$ satisfying supp $x \cap \operatorname{supp} y=\varnothing$ (see also [11]). Moreover, $\phi$ is norm attainable, i.e. there exists $x_{0} \in l^{\infty}$ such that $\left\langle\phi, x_{0}\right\rangle=\left\|x_{0}\right\|=1$ (cf. [2]). Define

$$
\|y\|_{1}=\max \left(\|y\|_{l^{\infty}}, 2|\langle\phi, y\rangle|\right), \quad y \in l^{\infty} .
$$

It is easy to see that $\|\cdot\|_{1}$ is an equivalent norm on $l^{\infty}$. Clearly, $\left(l^{\infty},\|\cdot\|_{1}\right) \in A M F$ and $\|\cdot\|_{1}$ is not order semi-continuous. Noticing that $\left\|x_{0}\right\|_{1}=2$ and $\left\|x_{0}\right\|_{l \infty}=1$, in virtue of Theorem 3.3 we get that $x_{0}$ is a smooth point in $\left(l^{\infty},\|\cdot\|_{1}\right)$, but $x_{0}$ cannot be decomposed by any atom $e \in \Sigma$ satisfying $\left\|x_{0} \chi_{e}\right\|=2$ and $\left\|x_{0} \chi_{\Omega \backslash e}\right\|<2$.

Example 3.6. Let $\Omega=\bigcup_{n \geq 1}\left[1 / 2^{2 n+1}, 1 / 2^{2 n}\right] \cup\{0\}$. It is known that $x \in C(\Omega)$ is a smooth point if and only if $x$ is a peak function. Let

$$
x(t)= \begin{cases}1, & x=0, \\ 1-1 / n, & x \in\left[1 / 2^{2 n+1}, 1 / 2^{2 n}\right] .\end{cases}
$$

It is easy to see that $x$ is a peak function. So $x$ is a smooth point. But $x$ cannot be decomposed by an atom of norm one and an element of norm less one. 


\section{The SMALlEST SUPPORT SEMI-NORM AT A POINT IN $A M F$ SPACES}

Definition 4.1. Let $X$ be a normed linear space and $x \in X \backslash\{0\}$. We say that a semi-norm $p(\cdot)$ is a support semi-norm at $x$ if $p(x)=\|x\|$ and $|\langle f, y\rangle| \leq p(y)$ holds for all $y \in X$ and $f \in S_{x}^{*}$. Moreover, if for any support semi-norm $q(\cdot)$ at $x$, we have $p(y) \leq q(y)$ for all $y \in X$, then we say that $p(\cdot)$ is the smallest support semi-norm at $x$.

One can easily verify that if $p(\cdot)$ is the smallest support semi-norm at $x$, then $p(y)=\sup \left\{|\langle f, y\rangle|: f \in S_{x}^{*}\right\}$.

Let $X \in A M F$. For any $x, y \in X$, let

$$
\begin{aligned}
& \Omega_{x, y}^{+}=\{t \in \Omega: x(t) y(t)>0\}, \\
& \Omega_{x, y}^{-}=\{t \in \Omega: x(t) y(t)<0\} .
\end{aligned}
$$

We denote by $E_{x, y}$ the set $\left\{e_{1}, \ldots, e_{4}\right\}$ of pairwise disjoint and $\mu$-measurable subsets of $\Omega$ such that $\Omega_{x, y}^{+}=e_{1} \cup e_{2}$ and $\Omega_{x, y}^{-}=e_{3} \cup e_{4}$, namely

$$
E_{x, y}=\left\{e_{j}: \Omega_{x, y}^{+}=e_{1} \cup e_{2}, \Omega_{x, y}^{-}=e_{3} \cup e_{4} ; e_{1} \cap e_{2}=e_{3} \cap e_{4}=\varnothing\right\},
$$

and denote by $\mathcal{E}_{x, y}$ the set of all decompositions $E_{x, y}$. If $\max \left(\left\|x \chi_{\Omega_{x, y}^{+}}\right\|,\left\|x \chi_{\Omega_{x, y}^{-}}\right\|\right)=$ $\|x\|$, then there exists some $e_{j} \in E_{x, y}$ such that $\left\|x \chi_{e_{j}}\right\|=\|x\|$. Now we define the following functionals:

$$
p_{x}(y)= \begin{cases}\inf _{E_{x, y} \in \mathcal{E}_{x, y}} \max _{\substack{e_{j} \in E_{x, y} \\\left\|x \chi_{e_{j}}\right\|=\|x\|}}\left\|y \chi_{e_{j}}\right\| & \text { if } \max \left(\left\|x \chi_{\Omega_{x, y}^{+}}\right\|,\left\|x \chi_{\Omega_{x, y}^{-}}\right\|\right)=\|x\|, \\ 0 & \text { if } \max \left(\left\|x \chi_{\Omega_{x, y}^{+}}\right\|,\left\|x \chi_{\Omega_{x, y}^{-}}\right\|\right)<\|x\|\end{cases}
$$

and

$$
r_{x}(y)= \begin{cases}\inf _{E_{x, y} \in \mathcal{E}_{x, y}} \underset{\substack{\operatorname{ess} \sup \\ t \in e_{j} \in E_{x, y} \\\left\|x \chi_{e_{j}}\right\|=\|x\|}}{\ln \|x\| \frac{|y(t)|}{|x(t)|}} & \text { if } \max \left(\left\|x \chi_{\Omega_{x, y}^{+}}\right\|,\left\|x \chi_{\Omega_{x, y}^{-}}\right\|\right)=\|x\|, \\ 0 & \text { if } \max \left(\left\|x \chi_{\Omega_{x, y}^{+}}\right\|,\left\|x \chi_{\Omega_{x, y}^{-}}\right\|\right)<\|x\|,\end{cases}
$$

where $\operatorname{ess}_{\sup _{t \in E}} z(t)=\inf _{E_{0} \subset E, \mu\left(E_{0}\right)=0} \sup _{t \in E \backslash E_{0}} z(t)$.

Theorem 4.2. Let $X \in A M F$ and let $x \in X \backslash\{0\}$. Then we have

(i) $p_{x}(y)=r_{x}(y)$ for all $y \in X$;

(ii) $p_{x}(\cdot)$ is the smallest support semi-norm at $x$.

Proof. (i) It suffices to consider the case $\max \left(\left\|x \chi_{\Omega_{x, y}^{+}}\right\|,\left\|x \chi_{\Omega_{x, y}^{-}}\right\|\right)=\|x\|$. Suppose that $\left\|x \chi_{e_{i}}\right\|=\|x\|$ and $\operatorname{ess} \sup _{t \in e_{i}}\|x\||y(t)| /|x(t)|=A$ for some $e_{i} \in E_{x, y}$. It is easy to see that there exists $e_{0} \subset e_{i}$ such that $\mu\left(e_{0}\right)=0$ and $\sup _{t \in e_{i} \backslash e_{0}}\|x\||y(t)| /|x(t)|=$ $A$. $t \in e_{i} \backslash e_{0}$ implies that $\|x\||y(t)| \leq A|x(t)|$. So we have $\|x\|\left\|y \chi_{e_{i}}\right\| \leq A\left\|x \chi_{e_{i}}\right\|=$ $A\|x\|$, i.e. $\left\|y \chi_{e_{i}}\right\| \leq A$. Hence it follows that $p_{x}(y) \leq r_{x}(y)$.

Conversely, for any $\varepsilon>0$, there exists $E_{x, y} \in \mathcal{E}_{x, y}$ such that

$$
\max _{\substack{e_{j} \in E_{x, y} \\\left\|x \chi_{e_{j}}\right\|=\|x\|}}\left\|y \chi_{e_{j}}\right\| \leq p_{x}(y)+\varepsilon
$$

Put

$$
e_{k}^{1}=\left\{\begin{array}{ll}
\left\{t \in e_{k}:\|x\||y(t)| \leq(1+\varepsilon)\left\|y \chi_{e_{k}}\right\||x(t)|\right\} & \text { if }\left\|x \chi_{e_{k}}\right\|=\|x\|, \\
\varnothing & \text { if }\left\|x \chi_{e_{k}}\right\|<\|x\|,
\end{array} \quad e_{k}^{2}=e_{k} \backslash e_{k}^{1} .\right.
$$


We have $\left\|x \chi_{e_{k}^{2}}\right\|<\|x\|$. In fact, if $\left\|x \chi_{e_{k}^{2}}\right\|=\|x\|$, then

$$
e_{k}^{2}=\left\{t \in e_{k}:\|x\||y(t)|>(1+\varepsilon)\left\|y \chi_{e_{k}}\right\||x(t)|\right\} .
$$

This leads to

$$
\|x\|\left\|y \chi_{e_{k}^{2}}\right\| \geq(1+\varepsilon)\left\|y \chi_{e_{k}}\right\|\left\|x \chi_{e_{k}^{2}}\right\|=(1+\varepsilon)\left\|y \chi_{e_{k}}\right\|\|x\| .
$$

A contradiction. Now we construct a new decomposition $E_{x, y}^{\prime}=\left\{\sigma_{i}: 1 \leq i \leq 4\right\}$ by letting

$$
\sigma_{1}=\bigcup_{k=1,2} e_{k}^{1}, \quad \sigma_{2}=\Omega_{x, y}^{+} \backslash \sigma_{1}, \quad \sigma_{3}=\bigcup_{k=3,4} e_{k}^{1}, \quad \sigma_{4}=\Omega_{x, y}^{-} \backslash \sigma_{3} .
$$

It is easy to see that $E_{x, y}^{\prime} \in \mathcal{E}_{x, y}$. From the construction of $\sigma_{i}$ we see that $\max _{i=1,3}\left\|x \chi_{\sigma_{i}}\right\|=\|x\|$ and $\max _{i=2,4}\left\|x \chi_{\sigma_{i}}\right\|<\|x\|$. Thus

$$
\begin{aligned}
\sup _{\substack{t \in \sigma_{i} \in E_{x, y}^{\prime} \\
\left\|x \chi_{\sigma_{i}}\right\|\|\| x \|}}\|x\| \frac{|y(t)|}{|x(t)|} & =\sup _{t \in \sigma_{1} \cup \sigma_{3}}\|x\| \frac{|y(t)|}{|x(t)|} \\
& \leq(1+\varepsilon) \max _{\substack{e_{i} \in E_{x, y} \\
\left\|x \chi_{e_{i}}\right\|=\|x\|}}\left\|y \chi_{e_{i}}\right\| \\
& \leq(1+\varepsilon)\left(p_{x}(y)+\varepsilon\right) .
\end{aligned}
$$

Since $\varepsilon>0$ is arbitrary, this means that $r_{x}(y) \leq p_{x}(y)$. The proof of (i) is complete.

(ii) First, we shall prove that $|\langle f, y\rangle| \leq p_{x}(y)$ for any $f \in S_{x}^{*}$ and $y \in X$. Assume for the contrary that there exist $f \in S_{x}^{*}$ and $y \in X$ such that $|\langle f, y\rangle|>p_{x}(y)$. In view of Corollaries 2.3 and 2.4, we have $\max \left(\left\|x \chi_{\Omega_{x, y}^{+}}\right\|,\left\|x \chi_{\Omega_{x, y}^{-}}\right\|\right)=\|x\|$. It follows from the definition of $p_{x}(\cdot)$ that there exists a decomposition

$$
E_{x, y}=\left\{e_{i}: \Omega_{x, y}^{+}=e_{1} \cup e_{2}, \Omega_{x, y}^{-}=e_{3} \cup e_{4} ; e_{1} \cap e_{2}=e_{3} \cap e_{4}=\varnothing\right\}
$$

such that

$$
|\langle f, y\rangle|>\max _{\substack{e_{j} \in E_{x, y} \\\left\|x \chi_{e_{j}}\right\|=\|x\|}}\left\|y \chi_{e_{j}}\right\| .
$$

Suppose that $e_{k} \in E_{x, y}$ satisfying $\left\|x \chi_{e_{k}}\right\|=\|x\|$ and

$$
\left\|y \chi_{e_{k}}\right\|=\max _{\substack{e_{j} \in E_{x, y} \\\left\|x \chi_{e_{j}}\right\|=\|x\|}}\left\|y \chi_{e_{j}}\right\| .
$$

We have $\mid\langle f, y\rangle>\left\|y \chi_{e_{k}}\right\|$. Let

$$
I_{1}=\left\{i:\left\|x \chi_{e_{i}}\right\|=\|x\|\right\}, \quad I_{2}=\left\{i:\left\|x \chi_{e_{i}}\right\|<\|x\|\right\} .
$$

In virtue of Corollaries 2.3 and 2.4 we have

$$
\left\langle f, y \chi_{\Omega \backslash\left(\Omega_{x, y}^{+} \cup \Omega_{x, y}^{-}\right)}\right\rangle=0 \quad \text { and } \quad\left\langle f, y \chi_{\cup_{i \in I_{2}} e_{i}}\right\rangle=0 .
$$

Therefore

$$
\begin{aligned}
|\langle f, y\rangle| & =\left|\left\langle f, y \chi_{\Omega_{x, y}^{+}}\right\rangle+\left\langle f, y \chi_{\Omega_{x, y}^{-}}\right\rangle\right| \\
& =\left|\left\langle f, y \chi_{\Omega_{x, y}^{+} \cap\left(\bigcup_{i \in I_{1}} e_{i}\right)}\right\rangle+\left\langle f, y \chi_{\Omega_{x, y}^{-} \cap\left(\bigcup_{i \in I_{1}} e_{i}\right)}\right\rangle\right| \\
& \leq\left\|y \chi_{\bigcup_{i \in I_{1}} e_{i}}\right\|=\left\|y \chi_{e_{k}}\right\| .
\end{aligned}
$$

This is a contradiction

Finally, we prove that $p_{x}(\cdot)$ is the smallest support semi-norm at $x$, i.e. $p_{x}(y)=$ $\sup \left\{|\langle f, y\rangle|: f \in S_{x}^{*}\right\}$ for any $y \in X$. If this equality does not hold, then there exist 
$\delta>0$ and $y \in X$ such that for any $f \in S_{x}^{*},|\langle f, y\rangle| \leq p_{x}(y)-\delta$. It is easy to see that $p_{x}(y)>0$. It follows that $\max \left(\left\|x \chi_{\Omega_{x, y}^{+}}\right\|,\left\|x \chi_{\Omega_{x, y}^{-}}\right\|\right)=\|x\|$. Let $E_{x, y} \in \mathcal{E}_{x, y}$ and

$$
E_{x, y}=\left\{e_{i}: \Omega_{x, y}^{+}=e_{i} \cup e_{2}, \Omega_{x, y}^{-}=e_{3} \cup e_{4} ; e_{1} \cap e_{2}=e_{3} \cap e_{4}=\varnothing\right\} .
$$

Take an arbitrary $\varepsilon>0$. Let

$$
e_{j \varepsilon}=\left\{t \in e_{j}:(1-\varepsilon) p_{x}(y)|x(t)| \leq\|x\||y(t)|\right\} \quad \text { if }\left\|x \chi_{e_{j}}\right\|=\|x\| .
$$

It follows that there exists at least one $e_{j \varepsilon}$ such that $\left\|x \chi_{e_{j \varepsilon}}\right\|=\|x\|$. Indeed, in the opposite case it would deduce that

$$
\max _{\substack{\sigma_{j} \in E_{x, y}^{\prime} \\\left\|x \chi_{\sigma_{j}}\right\|=\|x\|}}\left\|y \chi_{\sigma_{j}}\right\|<(1-\varepsilon) p_{x}(y),
$$

where $E_{x, y}^{\prime}=\left\{\sigma_{j}: 1 \leq j \leq 4\right\}$,

$$
\begin{array}{ll}
\sigma_{1}=\bigcup_{j \in\{1,2\}}\left\{e_{j \varepsilon}:\left\|x \chi_{e_{j}}\right\|=\|x\|\right\}, & \sigma_{2}=\Omega_{x, y}^{+} \backslash \sigma_{1}, \\
\sigma_{3}=\bigcup_{j \in\{3,4\}}\left\{e_{j \varepsilon}:\left\|x \chi_{e_{j}}\right\|=\|x\|\right\}, & \sigma_{4}=\Omega_{x, y}^{-} \backslash \sigma_{3},
\end{array}
$$

and $\sigma_{1}$ and $\sigma_{3}$ take empty sets if $\left\|x \chi_{e_{j}}\right\|<\|x\|$ for $j=1,2$ and for $j=3,4$ respectively. This is a contradiction. Let $f_{\varepsilon}$ be a support functional at $x \chi_{e_{j \varepsilon}}$. In view of Corollary 2.3 one can easily verify that $f_{\varepsilon}$ is also a support functional at $x$. So we have $\left\langle f_{\varepsilon}, y \chi_{\Omega \backslash e_{j \varepsilon}}\right\rangle=0$. Moreover, $t \in e_{j \varepsilon}$ implies that $y(t)$ has the same symbol as $x(t)$. Therefore,

$$
\begin{aligned}
\left|\left\langle f_{\varepsilon}, y\right\rangle\right| & =\left|\left\langle f_{\varepsilon}, y \chi_{e_{j \varepsilon}}\right\rangle\right| \\
& \geq\left|\left\langle f_{\varepsilon}, x \chi_{e_{j \varepsilon}}\right\rangle\right|(1-\varepsilon) p_{x}(y) /\|x\| \\
& =(1-\varepsilon) p_{x}(y) .
\end{aligned}
$$

Since $\varepsilon>0$ is arbitrary, this means that $\sup _{f \in S_{x}^{*}}|\langle f, y\rangle| \geq p_{x}(y)$. This contradicts to the inequality $\sup _{f \in S_{x}^{*}}|\langle f, y\rangle| \leq p_{x}(y)-\delta$. This ends the proof.

\section{ACKNOWLEDGEMENTS}

The first author is supported in part by the Special Doctoral Research Foundation of Hebei Province in China; the second author is supported in part by the Natural Science Foundation in China.

The authors would like to give their thanks to Professor A. R. Schep and the referee for their valuable comments and suggestions.

\section{REFERENCES}

1. S. Chen, H. Hudzik and A. Kaminska, Support functionals and smooth points in Orlicz function spaces equipped with the Orlicz norm, Math. Japonica 39 (1994), 271-279. MR 95f:46039

2. S. Chen and H. Sun, Weak convergence and weak compactness in abstract $M$ spaces, Proc. Amer. Math. Soc. 123 (1995), 1441-1447. MR 96b:46020

3. H. Hudzik and Y. N. Ye, Support functionals and smoothness of Musielak-Orlicz spaces endowed with the Luxemburg norm, Comment. Math. Univ. Carolinae 31 (1990), 661-684. MR 92g:46008;CMP 94:17

4. E. de Jonge, Representation of linear functionals on a class of normed Köthe spaces, J. Funct. Anal. 23 (1976), 119-134. MR 54:11045

5. S. Kakutani, Concrete representations of abstract $M$ spaces, Ann. of Math. 42 (1941), 9941024. MR 3:205g 
6. L. V. Kantorovich and G. B. Akilov, Functional Analysis, Izdat. Nauka, Moscow, 1977. MR 58:23465

7. J. Lindenstrauss and L. Tzafriri, Classical Banach Spaces II, Springer-Verlag, 1979. MR 81c: 46001

8. W. A. Luxemburg and A. C. Zaanen, Riesz Spaces I, North Holland Math. Library, 1971. MR 58:23483

9. Baoxiang Wang, Support functional and its applications of Orlicz spaces, Comment. Math. 33 (1993), 185-192. MR 94m:46052

10. Baoxiang Wang and Meilan $\mathrm{Xu}$, On some applications of support functionals of Orlicz spaces, Comment. Math. 35 (1995), 235-244. MR 97a:46038

11. B. X. Wang and T. F. Wang, The characteristic of weak convergence in a class of Banach sequence lattices, Collect. Math. 48 (1997), 799-807. CMP 98:08

12. Xintai Yu, Geometry of Banach Spaces, Shanghai, 1986.

13. A. C. Zaanen, Riesz Spaces II, North Holland Math. Library, 1983. MR 86b:46001

Department of Mathematics, Hebei University, Baoding 071002, People's Republic of CHINA

E-mail address: math@nic.hbu.edu.cn

Department of Mathematics, Harbin University of Science and Technology, Harbin, 150080, People's Republic of China 